\title{
ESTIMATES FOR PARAMETRIC MARCINKIEWICZ INTEGRALS ON MUSIELAK-ORLICZ HARDY SPACES
}

\author{
Xiong Liu, Baode Li, Xiaoli QiU and Bo Li
}

\begin{abstract}
Let $\varphi: \mathbb{R}^{n} \times[0, \infty) \rightarrow[0, \infty)$ satisfy that $\varphi(x, \cdot)$, for any given $x \in \mathbb{R}^{n}$, is an Orlicz function and $\varphi(\cdot, t)$ is a Muckenhoupt $A_{\infty}$ weight uniformly in $t \in(0, \infty)$. The MusielakOrlicz Hardy space $H^{\varphi}\left(\mathbb{R}^{n}\right)$ generalizes both of the weighted Hardy space and the Orlicz Hardy space and hence has a wide generality. In this paper, the authors first prove the completeness of both of the Musielak-Orlicz space $L^{\varphi}\left(\mathbb{R}^{n}\right)$ and the weak Musielak-Orlicz space $W L^{\varphi}\left(\mathbb{R}^{n}\right)$. Then the authors obtain two boundedness criterions of operators on Musielak-Orlicz spaces. As applications, the authors establish the boundedness of parametric Marcinkiewicz integral $\mu_{\Omega}^{\rho}$ from $H^{\varphi}\left(\mathbb{R}^{n}\right)$ to $L^{\varphi}\left(\mathbb{R}^{n}\right)$ (resp. $W L^{\varphi}\left(\mathbb{R}^{n}\right)$ ) under weaker smoothness condition (resp. some Lipschitz condition) assumed on $\Omega$. These results are also new even when $\varphi(x, t):=\phi(t)$ for all $(x, t) \in \mathbb{R}^{n} \times[0, \infty)$, where $\phi$ is an Orlicz function.
\end{abstract}

Mathematics subject classification (2010): Primary 42B20, Secondary 42B30, 46E30.

Keywords and phrases: Marcinkiewicz integral, Muckenhoupt weight, Musielak-Orlicz function, Hardy space.

\section{REFERENCES}

[1] Ali Akbulut, OKan KuZU, Marcinkiewicz integrals associated with Schrödinger operator on generalized Morrey spaces, J. Math. Inequal. 8 (2014), no. 4, 791-801.

[2] The Anh Bui, Jun Cao, Luong Dang Ky, Dachun Yang, Sibei Yang, Musielak-OrliczHardy spaces associated with operators satisfying reinforced off-diagonal estimates, Anal. Geom. Metr. Spaces 1 (2013), 69-129.

[3] Jun Cao, Der-Chen Chang, Dachun Yang, Sibei Yang, Riesz transform characterizations of Musielak-Orlicz Hardy spaces, Trans. Amer. Math. Soc. 368 (2016), no. 10, 6979-7018.

[4] LARS DiEnING, Maximal function on Musielak-Orlicz spaces and generalized Lebesgue spaces, Bull. Sci. Math. 129 (2005), no. 8, 657-700.

[5] Lars Diening, Peter A. Hästö, Svetlana Roudenko, Function spaces of variable smoothness and integrability, J. Funct. Anal. 256 (2009), no. 6, 1731-1768.

[6] Xingya Fan, Jianxun He, Baode Li, Dachun Yang, Real-variable characterizations of anisotropic product Musielak-Orlicz Hardy spaces, Sci. China Math. 60, 11 (2017), 2093-2154.

[7] Vagif S. Guliyev, Ali Akbulut, Vugar H. Hamzayev, Okan Kuzu, Commutators of Marcinkiewicz integrals associated with Schrödinger operator on generalized weighted Morrey spaces, J. Math. Inequal. 10 (2016), no. 4, 947-970.

[8] Loukas Grafakos, Classical Fourier Analysis, Second edition, Graduate Texts in Mathematics Vol. 249 (Springer, New York, 2008).

[9] Loukas Grafakos, Modern Fourier Analysis, Second edition, Graduate Texts in Mathematics Vol. 250 (Springer, New York, 2009).

[10] LARS HÖRMANDER, Estimates for translation invariant operators in $L^{p}$ spaces, Acta Math. 104 (1960), 93-140. 
[11] Shaoxiong Hou, Dachun Yang, Sibei Yang, Lusin area function and molecular characterizations of Musielak-Orlicz. Hardy spaces and their applications, Commun. Contemp. Math. 15 (2013), no. 6, 1350029, $37 \mathrm{pp}$.

[12] Svante Janson, Generalizations of Lipschitz spaces and an application to Hardy spaces and bounded mean oscillation, Duke Math. J. 47 (1980), no. 4, 959-982.

[13] Renjin JiAng, DACHUn YAng, New Orlicz-Hardy spaces associated with divergence form elliptic operators, J. Funct. Anal. 258 (2010), no. 4, 1167-1224.

[14] Raymond L. Johnson, Christoph Johannes Neugebauer, Homeomorphisms preserving $A_{p}$, Rev. Mat. Iberoamericana 3 (1987), no. 2, 249-273.

[15] Douglas S. Kurtz, Richard L. WheEden, Results on weighted norm inequalities for multipliers, Trans. Amer. Math. Soc. 255 (1979), 343-362.

[16] LuOng DANG Ky, New Hardy spaces of Musielak-Orlicz type and boundedness of sublinear operators, Integral Equations Operator Theory 78 (2014), no. 1, 115-150.

[17] BaOde Li, Xingya Fan, Dachun Yang, Littlewood-Paley characterizations of anisotropic Hardy spaces of Musielak-Orlicz type, Taiwanese J. Math. 19 (2015), no. 1, 279-314.

[18] Baode Li, Xingya Fan, Zunwei Fu, Dachun YAng, Molecular characterization of anisotropic Musielak-Orlicz Hardy spaces and their applications, Acta Math. Sin. (Engl. Ser.) 32 (2016), no. 11, 1391-1414.

[19] JinXia Li, Ruirui Sun, BaOde Li, Anisotropic interpolation theorems of Musielak-Orlicz type, J. Inequal. Appl. 2016, 2016: 243.

[20] Yiyu LiAng, Jizheng HuAng, Dachun YANG, New real-variable characterizations of MusielakOrlicz Hardy spaces, J. Math. Anal. Appl. 395 (2012), no. 1, 413-428.

[21] Yiyu Liang, Dachun Yang, Musielak-Orlicz Campanato spaces and applications, J. Math. Anal. Appl. 406 (2013), no. 1, 307-322.

[22] Yiyu Liang, Dachun Yang, Renjin Jiang, Weak Musielak-Orlicz Hardy spaces and applications, Math. Nachr. 289 (2016), no. 5-6, 634-677.

[23] Chin-Cheng Lin, Ying-Chieh Lin, $H_{\omega}^{p}-L_{\omega}^{p}$ boundedness of Marcinkiewicz integral, Integral Equations Operator Theory 58 (2007), no. 1, 87-98.

[24] Bo Li, Minfeng LiaO, BaOde Li, Boundedness of Marcinkiewicz integrals with rough kernels on Musielak-Orlicz Hardy spaces, J. Inequal. Appl. 2017, 2017: 228.

[25] J. Marcinkiewicz, Sur quelques intégrales du type de Dini, Annales de la Société Polonaise de Mathématiques 17 (1938), 42-50.

[26] Xinfeng Shi, Yinsheng Jiang, Weighted boundedness of parametric Marcinkiewicz integral and higher order commutator, Anal. Theory Appl. 25 (2009), no. 1, 25-39.

[27] Rolewicz Stefan, Metric Linear Spaces, Second edition, (PWN-Polish Scientific Publishers, Warsaw, 1984).

[28] Elias M. Stein, On the functions of Littlewood-Paley, Lusin, and Marcinkiewicz, Trans. Amer. Math. Soc. 88 (1958), 430-466.

[29] Jan-Olov Strömberg, Alberto Torchinsky, Weighted Hardy spaces, Lecture Notes in Mathematics Vol. 1381 (Springer-Verlag, Berlin, 1989).

[30] Jan-Olov Strömberg, Richard L. Wheeden, Fractional integrals on weighted $H^{p}$ and $L^{p}$ spaces, Trans. Amer. Math. Soc. 287 (1985), no. 1, 293-321.

[31] HuA WAng, Parametric Marcinkiewicz integrals on the weighted Hardy and weak Hardy spaces, J. Math. Inequal. 10 (2016), no. 2, 373-391.

[32] Dachun Yang, Yiyu Liang, Luong Dang Ky, Real-Variable Theory of Musielak-Orlicz Hardy spaces, Lecture Notes in Mathematics Vol. 2182 (Springer, Cham, 2017). 